\title{
The Impact of Neurophysiological Intraoperative Monitoring during Spinal Cord and Spine Surgery: A Critical Analysis of 121 Cases
}

Tarik Ibrahim ${ }^{1}$, Oliver Mrowczynski ${ }^{1}$, Omar Zalatimo ${ }^{2}$, Vernon Chinchilli ${ }^{3}$, Jonas Sheehan ${ }^{4}$, Robert Harbaugh ${ }^{1}$, Elias Rizk ${ }^{1}$

1. Department of Neurosurgery, Penn State Hershey Medical Center 2. Department of Neurosurgery, Lifebridge Health Sinai Hospital 3. Public Health Sciences, Penn State University College of Medicine 4. Neurosurgery, Holy spirit hospital

Corresponding author: Elias Rizk, erizk@hmc.psu.edu

\begin{abstract}
Neuromonitoring has been utilized during spinal surgery to assess the function of the spinal cord in an effort to prevent intraoperative injury. Although its use is widespread, no clear benefit has been demonstrated. Our goal in this study was to interrogate the value of intraoperative neuromonitoring in decreasing the severity and rate of neurological injury during and after spinal surgery. Here we describe our experience of 121 patients who underwent spinal cord procedures with the combination of intraoperative neuromonitoring, to determine its ability to detect neurological changes and the specificity and sensitivity in this setting. The data for the 121 patients who underwent neurophysiological monitoring during various spinal procedures was collected retrospectively. The patients were classified into one of four groups according to the findings of intraoperative monitoring and the clinical outcomes on postoperative neurological exam. Intraoperative monitoring was evaluated for its specificity, sensitivity, and predictive value. In our cohort of 121 patients, the use of intraoperative neuromonitoring had a low sensitivity, which may produce an excessive number of false negatives. Based on these findings, neuromonitoring seems to have a poor positive predictive value and is thus an inappropriate test to prevent harm to patients.
\end{abstract}

Received 10/16/2017

Review began 10/23/2017 Review ended 10/30/2017 Published 11/19/2017

๑) Copyright 2017

Ibrahim et al. This is an open access article distributed under the terms of the Creative Commons Attribution License CC-BY 3.0., which permits unrestricted use, distribution, and reproduction in any medium, provided the original author and source are credited.
Categories: Neurosurgery

Keywords: intraoperative monitoring, spinal surgery, neuromonitoring, spine surgery

\section{Introduction}

Neuromonitoring has long been used during spinal surgery to assess the function of the spinal cord in an effort to prevent intraoperative injury [1]. Although its use is widespread, no clear benefit has been demonstrated. Some evidence suggests that intraoperative monitoring is a cost-effective component of spinal surgery [2] that provides critical information enabling the surgical team to give the patient optimal postoperative neurologically functional outcomes. There are other reports in the literature that demonstrate a failure of neuromonitoring to predict postoperative outcome [3-8]. The exact efficacy of the utilization of intraoperative neuromonitoring is not well understood. Patient outcomes and improvement following surgical procedures is paramount, and testing whether intraoperative neuromonitoring aids in this regard is critical. Our goal in this study was to interrogate the value of intraoperative neuromonitoring to decrease the severity and rate of neurological injury during and after spinal surgery. Here we describe our experience of 121 patients who underwent spinal cord procedures utilizing intraoperative neuromonitoring, to determine its ability to be specific and sensitive for the accurate diagnosis of neurological deficit in this setting.

\section{Materials And Methods}

From January to December 2006, 121 patients (61 male, 60 female) underwent spinal surgery with multimodality intraoperative neurophysiologic monitoring. The ages ranged from one month old to 83 years old, with a mean age of 41.4 years. The cases were categorized into cervical, thoracic, and lumbar regions (Table 1). 


\section{Cureus}

\begin{tabular}{|c|c|c|}
\hline Spinal Region & Operation & $\mathbf{N}$ \\
\hline \multirow[t]{7}{*}{ Cervical (57) } & Halo adjustment & 1 \\
\hline & Corpectomy & 2 \\
\hline & Anterior fusion & 3 \\
\hline & Intramedullary tumor resection & 2 \\
\hline & Laminectomy & 9 \\
\hline & Posterior fusion & 8 \\
\hline & ACDF & 32 \\
\hline \multirow[t]{7}{*}{ Thoracic (30) } & Kyphoplasty & 1 \\
\hline & Revision of spinal rods & 1 \\
\hline & Anterior fusion & 1 \\
\hline & Corpectomy & 1 \\
\hline & Posterior fusion & 5 \\
\hline & Laminectomy & 7 \\
\hline & Thoraco-lumbar fusion & 14 \\
\hline \multirow[t]{7}{*}{ Lumbar (34) } & Laminectomy & 1 \\
\hline & Discectomy & 1 \\
\hline & Pars repair & 1 \\
\hline & Radical tumor resection & 1 \\
\hline & Intramedulalry tumor resection & 3 \\
\hline & Osteotomy/revision/lengthening of spinal rods & 5 \\
\hline & Posterior fusion & 22 \\
\hline \multicolumn{3}{|l|}{ al (121) } \\
\hline
\end{tabular}

\section{TABLE 1: Summary of operative procedures}

Fifty-seven cervical operations and seven types of cervical procedures were performed. These included halovest adjustment, anterior cervical discectomy and fusion (ACDF) with instrumentation, corpectomy and fusion with instrumentation, anterior fusion with instrumentation, posterior fusion with instrumentation (one of which extended to the thoracic spine), laminectomy for decompression, and intramedullary tumor resection. Thirty thoracic procedures were performed, including posterior thoracolumbar fusions, posterior fusion with instrumentation, corpectomy and instrumented fusion, laminectomy for decompression, kyphoplasty, and revision of spinal instrumentation and fusion. Thirty-four lumbar procedures were done. These included laminectomy, discectomy, posterior fusion with instrumentation, pars interarticularis fracture open reduction and internal fixation, intramedullary tumor resection, tethered cord release, and osteotomy revision with lengthening of spinal rods.

Neurophysiological potentials were monitored continuously throughout surgery. Modalities varied upon indications and included the following (with percentage of patients receiving each in parentheses): somatosensory evoked potentials (SSEPs; 98.4\%), transcranial motor evoked potentials (TCMEPs; 86.3\%), electromyography (EMG; 90.2\%), train of four (TOF; 34.1\%), electroencephalography (EEG; 19.5\%), and 


\section{Recording protocol}

General anesthesia was induced in all cases with a mixture of propofol, fentanyl, and rocuronium. Anesthesia was thereafter maintained by propofol and fentanyl.

\section{SSEPS}

Median and ulnar nerve SSEPs (MN-SSEP and UN-SSEP, respectively) were elicited at the wrist via nerve stimulation for a duration of $300 \mathrm{msec}$ and approximately $25 \mathrm{~mA}$ intensity at a $4.76 \mathrm{~Hz}$ stimulation rate. Posterior tibial nerve SSEPs (PT-SSEPs) were elicited at the ankle by the same parameters used in the upper extremities. The stimulation rate was sometimes lowered to $3.1 \mathrm{~Hz}$ if the baseline amplitudes were low. Surface pad electrodes were used to stimulate the SSEPs. The SSEPs were recorded using subcutaneous needle electrodes.

The recording arrays for MN-SSEPs and UN-SSEPs were placed at C3'/C4' with the reference electrode Fpz, Cv5'-Fpz. A proximal, brachial plexus recording was taken at RErb's point - LErb's point or the reverse depending on the side of stimulation. For the PT-SSEPs, the recording arrays are C3'/C4'-Fpz, C3'-C4' (or reverse depending on the side of stimulation) and Cv5-Fpz.

A surface pad ground electrode was placed on the deltoid or trapezius. The resistance of all electrodes was below $5 \mathrm{kOhm}$ and all were within $2 \mathrm{kOhm}$ of each other. The time base was set to $100 \mathrm{msec}$, filter settings at $10-3 \mathrm{kHz}$, amplifier gain at $100 \mathrm{uV} /$ div and typically averaged between 200 and 300 trials.

\section{BAEPs, aka auditory brainstem response (ABR)}

The filter settings were 30-3k Hz with an amp gain of $10 \mathrm{uV} /$ div. We used EAR-3 foam insert earphones with a distal stimulus generator and an air tube conducting the stimulus. The initial stimulation intensity was 70 $\mathrm{dB}$ SL, but if an audiogram was not available, the typical starting range for normal hearing patients was 80 dB HL. Rare fraction clicks were used at a rate between 11.1 and $33.1 \mathrm{~Hz}$. Recording electrode arrays were $\mathrm{A} 1 / \mathrm{A} 2-\mathrm{Cz}^{\prime}$ and $\mathrm{A} 1-\mathrm{A} 2$ (or reverse depending on the stimulus side). Needle electrodes were placed in the ear canals. The time base was $20 \mathrm{msec}$ and 1000-2000 trials were averaged.

Stimulation, recording, and data processing were all done with the Cadwell Cascade (Cadwell Laboratories, Inc. WA, USA). The evoked potentials (EPs) were recorded and visually analyzed for the presence of the main peaks. For MN-SSEPs and UN-SSEPs, the cortical complex N20-P25 was identified. For the PT-SSEPs, the cortical complex of P37-N45 was analyzed. ABR waves I, III, and V were analyzed for presence or absence. Interpeak and I-V latencies were monitored. Wave $\mathrm{V}$ amplitude was also monitored. The baselines were collected post-induction, but pre-incision. Occasionally, if the patient was unstable, baselines were collected before the patient was positioned on the surgical table and then again after positioning. Data was then compared and the positioning was adjusted accordingly.

\section{Collection of data}

The criteria by which a significant neuromonitoring finding was defined were the following: MN-SSEPs: a reduction in amplitude by more than $50 \%$ in the cortical complex N20-P25 and/or an increase of the peak latency of N20 by more than $10 \%$ compared to the preoperative baseline; PT-SSEPs: a reduction in cortical complex P40-N50 amplitude of more than 50\% and/or an increase of P40 peak latency by more than $10 \%$ compared to the baseline value. Loss of Waves I, III or V in any combination was considered a pathological finding for BAEP. When a neuromonitoring abnormality was detected, the surgeon and anesthesiologist were immediately notified and the following possible sources were immediately explored: physiological irregularities, anesthetic changes, and surgical manipulation.

Every patient had a neurological examination performed postoperatively by a member of the neurosurgery team. All deficits were documented and compared to the preoperative examination to determine if neurological findings were present preoperatively or acquired during surgery. Deficits were re-evaluated at the patient's first outpatient visit following discharge and compared to the findings immediately following surgery. The neurological findings were correlated with intraoperative neuromonitoring changes. Based on this assessment, patients were assigned to one of four categories: false positive, true positive, false negative, and true negative. Patients who developed a neuromonitoring change that could not be corrected intraoperatively but who did not display any new neurological deficit postoperatively were designated as false positives. Patients who developed a neuromonitoring change intraoperatively and who demonstrated a new neurological deficit postoperatively were designated as true positives. In addition, a patient was designated as a true positive if an intraoperative neuromonitoring change occurred, the change was corrected intraoperatively, and the patient awoke without a new deficit. Patients who developed a new neurological deficit intraoperatively without a change in neuromonitoring were designated as false negatives. Finally, if no neuromonitoring changes occurred and the patient awoke without a new deficit, this was determined to be a true negative. 


\section{Results}

We describe the results of 121 patients who underwent spinal surgeries as described in Table 1 with intraoperative neuromonitoring (Figure $1 \mathrm{~A}$ ).

A

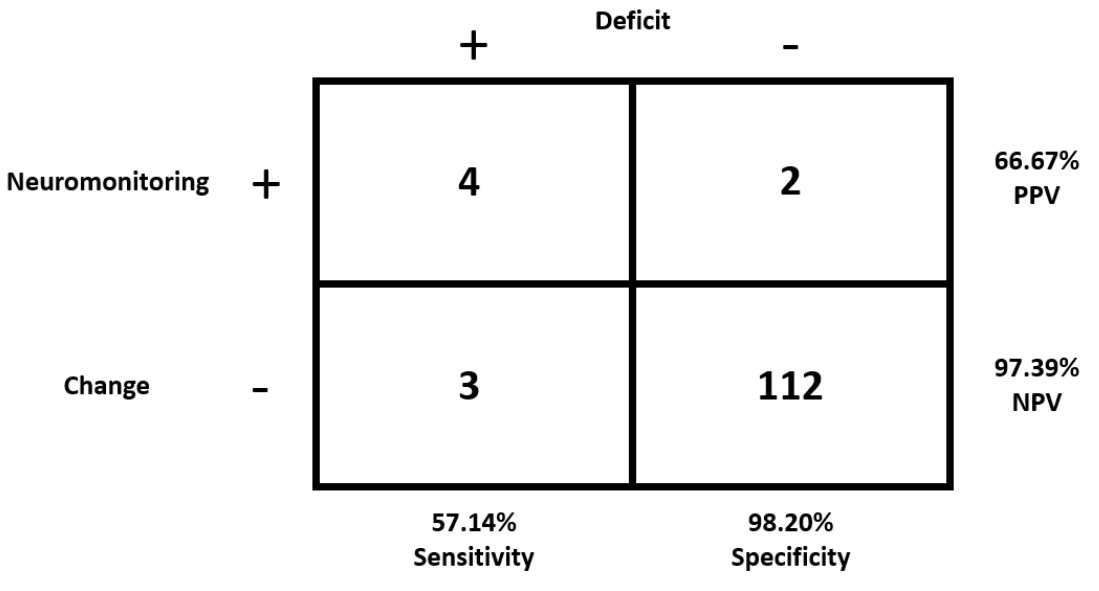

\begin{tabular}{|l|l|l|l|}
\hline & Neuromonitoring & Deficit? & Clinical Outcome \\
\hline False Negative & No change & UE grip strength & Resolved \\
\hline False Negative & No change & Iliopsoas strength & Resolved \\
\hline False Negative & No change & Decreased thigh sensation & Resolved \\
\hline
\end{tabular}

\section{FIGURE 1: Results of our analysis on the use of intraoperative monitoring during 121 spinal surgery cases}

(A) Out of 121 patients, seven had neurological deficits. Four of those patients were accurately diagnosed with neuromonitoring, while the other three had false negatives. Out of the 114 patients who did not have a neurological deficit, neuromonitoring accurately diagnosed the lack of deficits in 112 (Negative Predictive Value - NPV), while the other two had false positives (Positive Predictive Value - PPV). (B) The three patients who had clinically diagnosed neurological deficits in upper extremity (UE) grip strength, iliopsoas strength, and decreased thigh sensation were not accurately diagnosed by neuromonitoring.

Four out of seven patients who had a neurological deficit were able to be detected by neuromonitoring. Neuromonitoring was not able to detect the present deficits in the three other patients. This equates to a sensitivity of $57.14 \%$. Out of 114 patients who did not have a neurological deficit, neuromonitoring accurately diagnosed no deficits in 112 . The two other patients were falsely rendered as having deficits, when there were no clinical neurological deficits present, equating to a specificity of $98.20 \%$. The positive predicted value of this data is $66.67 \%$, while the negative predicted value is $97.39 \%$.

We further assessed this data with regard to the three patients who were deemed having false negative results. The three patients had clinically diagnosed neurological deficits (Figure 1B). The first patient had an upper extremity deficit of grip strength at their postoperative check that was resolved by the next day. Another patient had iliopsoas strength graded at $4 / 5$ immediately postoperatively, which improved to $5 / 5$ at follow-up in the outpatient clinic. The third patient woke from surgery with decreased sensation on the antero-lateral portion of the thigh, which persisted until the first postoperative clinic appointment. These deficits were not accurately diagnosed by neuromonitoring.

\section{Discussion}

Spinal surgery is a common procedure that has a risk of neurological deficit [9]. Damage to the spinal cord during a procedure can occur through stretch of the cord/nerve, hypoxia, or direct damage

[10]. Intraoperative neuromonitoring is a tool with the goal of providing patients with limited morbidities and optimal outcomes during and after surgery. The aim of neuromonitoring during an operation is to provide the surgeon with a real-time analysis of spinal cord function at a time when there is still a possibility to correct any possibility of morbidity [11]. Changes in intraoperative neuromonitoring measurements can be due to changes in arterial pressure, cardiopulmonary function, and spinal cord function [12]. Potentials can also be influenced by anesthetic regimen [3, 13-15], perfusion pressure [16-17], hypothermia [18], and hyperthermia [19]. Intraoperative neuromonitoring has been utilized in many contexts, including spine surgery, arteriovenous malformations, thyroid and parathyroid surgery, pediatric deformity correction surgery, epilepsy surgery, subarachnoid hemorrhage repair, and others. Although it has been used in the numerous contexts shown above, an obvious benefit of intraoperative neuromonitoring providing optimal 
One area highlighting this contention in the case of spinal surgery is spinal tumor resection. In a review by Scibilia, et al. on the use of intraoperative neuromonitoring in the specific scenario of spinal tumors, the authors came to the conclusion that neuromonitoring is a useful technology to aid in providing patients with adequate postoperative outcomes [20]. Other studies assessing the effect of neuromonitoring in spinal tumor surgery demonstrate that motor evoked potentials and multi-modal monitoring did not accurately provide predictive value for permanent functional deficits [21]. Significant changes occur most frequently in intramedullary lesions [22]. In such cases SSEPs have been found to have good sensitivity but poor specificity [23], and there are concerns that false positive changes during SSEP monitoring could also prematurely halt adequate surgical intervention [24]. Combining MEP with SSEP allows for a better prediction of motor outcome in spinal cord surgery. False positive results with MEPs have also occurred [2526]. Furthermore, MEPs are highly variable and very sensitive to the effect of anesthesia and muscle relaxant. This adds another variable to the prediction of motor deficits through neuromonitoring [18]. Wiedmayer, et al. reported that in more than $50 \%$ of the cases, the surgeon was not able to respond to a monitoring event [22].

To try and enhance the efficacy of intraoperative neuromonitoring, "checklists" are being developed that provide a methodology of utilizing this technology optimally during surgery [27-28]. These checklists describe what the surgeon, anesthesiologist, neurophysiologists, and technicians should perform to manage a significant alert given by the intraoperative neuromonitoring [27].

The clinical goal of any test is to provide a result with a high degree of sensitivity and specificity with the hope of improving patient care and outcomes. When either of these attributes are low, data interpretation is confounded. In the case of intraoperative neuromonitoring, a low sensitivity was found in the present study, which may produce an excessive number of false negatives. Other studies such as that done by May, et al. found intraoperative monitoring to have low specificity such that a physician may experience more false positives [3]. Both low sensitivity and low specificity can have detrimental effects on the surgery and adversely affect patient outcomes. Particularly in cases in which there is a high risk of neurological injury, a lack of detected neurophysiological change (false negative) may give the surgeon a false sense of security and encourage him to continue performing a potentially damaging progress. The absence of findings could lead a surgeon to go beyond the bounds of his clinical and surgical judgment, exposing the patient to greater danger and harm.

The authors acknowledge several limitations of the present study. First, all included data was collected and reported retrospectively, thus some publication bias may exist. Second, there was a small sample size of patients who did have neurological deficits. Further studies in a greater number of patients are necessary to determine the full extent of efficacy of intraoperative neuromonitoring in the context of spine surgery.

\section{Conclusions}

Our goal in this study was to interrogate the value of intraoperative neuromonitoring to decrease the severity and rate of neurological injury during and after spinal surgery. In our cohort of 121 patients, the use of intraoperative neuromonitoring had a low sensitivity, which may produce an excessive number of false negatives. Spine surgeons need to be aware of the low sensitivity and positive predictive value with neuromonitoring so that they rely more on their clinical and surgical judgement and interpret neuromonitoring with more scrutiny.

\section{Additional Information \\ Disclosures}

Human subjects: Consent was obtained by all participants in this study. Animal subjects: All authors have confirmed that this study did not involve animal subjects or tissue. Conflicts of interest: In compliance with the ICMJE uniform disclosure form, all authors declare the following: Payment/services info: All authors have declared that no financial support was received from any organization for the submitted work. Financial relationships: All authors have declared that they have no financial relationships at present or within the previous three years with any organizations that might have an interest in the submitted work. Other relationships: All authors have declared that there are no other relationships or activities that could appear to have influenced the submitted work.

\section{Acknowledgements}

Tarik Ibrahim is deceased and is included as a coauthor for the following contributions. Dr. Ibrahim was responsible for data collection and analysis, as well as drafting the manuscript. The co-authors would like to acknowledge his noteworthy contribution to this paper.

\section{References}

1. Nash CL Jr, Lorig RA, Schatzinger LA, Brown RH: Spinal cord monitoring during operative treatment of the 
spine. Clin Orthop Relat Res. 1977, 126:100-5.

2. Sala F, Dvorak J, Faccioli F: Cost effectiveness of multimodal intraoperative monitoring during spine surgery. Eur Spine J. 2007, 16:229-231. 10.1007/s00586-007-0420-0

3. May DM, Jones SI, Crockard HA: Somatosensory evoked potential monitoring in cervical surgery: identification of pre- and intraoperative risk factors associated with neurological deterioration. J Neurosurg. 1996, 85:566-573. 10.3171/ins.1996.85.4.0566

4. Pelosi L, Jardine A, Webb JK: Neurological complications of anterior spinal surgery for kyphosis with normal somatosensory evoked potentials (SEPs). J Neurol Neurosurg Psychiatry. 1999, 66:662-4.

10.1136/INNP.66.5.662

5. Jones SI, Buonamassa S, Crockard HA: Two cases of quadriparesis following anterior cervical discectomy, with normal perioperative somatosensory evoked potentials. J Neurol Neurosurg Psychiatry. 2003, 74:273-6. 10.1136/jnnp.74.2.273

6. Minahan RE, Sepkuty JP, Lesser RP, Sponseller PD, Kostuik JP: Anterior spinal cord injury with preserved neurogenic "motor" evoked potentials. Clin Neurophysiol. 2001, 112:1442-50. 10.1016/S13882457(01)00567-3

7. Zornow MH, Grafe MR, Tybor C, Swenson MR: Preservation of evoked potentials in a case of anterior spinal artery syndrome. Electroencephalogr Clin Neurophysiol. 1990, 77:137-9.

8. Lesser RP, Raudzens P, Lüders H, et al.: Postoperative neurological deficits may occur despite unchanged intraoperative somatosensory evoked potentials. Ann Neurol. 1986, 19:22-25. 10.1002/ana.410190105

9. Schwartz DM, Auerbach JD, Dormans JP, et al.: Neurophysiological detection of impending spinal cord injury during scoliosis surgery. J Bone Jt Surg. 2007, 89:2440-9.

10. Vitale MG, Moore DW, Matsumoto H, et al.: Risk factors for spinal cord injury during surgery for spinal deformity. J Bone Jt Surg Am. 2010, 92:64-71.

11. Devlin VJ, Schwartz DM: Intraoperative neurophysiologic monitoring during spinal surgery. J Am Acad Orthop Surg. 2007, 15:549-60.

12. Noonan KJ, Walker T, Feinberg JR, Nagel M, Didelot W, Lindseth R: Factors related to false- versus truepositive neuromonitoring changes in adolescent idiopathic scoliosis surgery. Spine (Phila Pa 1976). 2002, $27: 825-30$.

13. Friedman WA, Kaplan BL, Day AL, Sypert GW, Curran MT: Evoked potential monitoring during aneurysm operation: observations after fifty cases. Neurosurgery. 1987, 20:678-87.

14. Szalay EA, Carollo JJ, Roach JW: Sensitivity of spinal cord monitoring to intraoperative events . J Pediatr Orthop. 1986, 6:437-41.

15. Dawson EG, Sherman JE, Kanim LE, Nuwer MR: Spinal cord monitoring. Results of the Scoliosis Research Society and the European Spinal Deformity Society survey. Spine (Phila Pa 1976). 1991, 16:S361-4.

16. Sebastián C, Raya JP, Ortega M, Olalla E, Lemos V, Romero R: Intraoperative control by somatosensory evoked potentials in the treatment of cervical myeloradiculopathy. Eur Spine J. 1997, 6:316-23. 10.1007/BF01142677

17. Forbes HJ, Allen PW, Waller CS, Jones SJ, Edgar MA, Webb PJ, Ransford AO: Spinal cord monitoring in scoliosis surgery. Experience with 1168 cases. J Bone Joint Surg Br. 1991, 73:487-91.

18. Jones SJ, Harrison R, Koh KF, Mendoza N, Crockard HA: Motor evoked potential monitoring during spinal surgery: responses of distal limb muscles to transcranial cortical stimulation with pulse trains. Electroencephalogr Clin Neurophysiol. 1996, 100:375-383. 10.1016/0168-5597(96)95728-7

19. Kartush JM: Electroneurography and intraoperative facial monitoring in contemporary neurotology . Otolaryngol Neck Surg. 1989, 101:496-503. 10.1177/019459988910100416

20. Scibilia A, Terranova C, Rizzo V, et al.: Intraoperative neurophysiological mapping and monitoring in spinal tumor surgery: sirens or indispensable tools?. Neurosurg Focus. 2016, 41:E18. 10.3171/2016.5.FOCUS16141

21. Lakomkin N, Mistry AM, Zuckerman SL, et al.: Utility of intraoperative monitoring in the resection of spinal cord tumors: an analysis by tumor location and anatomical region. Spine (Phila Pa 1976). 2017, [Epub ahead of print]:10.1097/BRS.0000000000002300

22. Wiedemayer H, Fauser B, Sandalcioglu IE, Schäfer H, Stolke D: The impact of neurophysiological intraoperative monitoring on surgical decisions: a critical analysis of 423 cases. J Neurosurg. 2002, 96:25562. 10.3171/jns.2002.96.2.0255

23. Kearse LA, Brown EN, McPeck K: Somatosensory evoked potentials sensitivity relative to electroencephalography for cerebral ischemia during carotid endarterectomy. Stroke. 1992, 23:498-505. 10.1161/01.STR.23.4.498

24. Kearse LA Jr1, Lopez-Bresnahan M, McPeck K, Tambe V: Loss of somatosensory evoked potentials during intramedullary spinal cord surgery predicts postoperative neurologic deficits in motor function [corrected]. J Clin Anesth. 1993, 5:392-8.

25. Kothbauer KF, Deletis V, Epstein FJ: Motor-evoked potential monitoring for intramedullary spinal cord tumor surgery: correlation of clinical and neurophysiological data in a series of 100 consecutive procedures. Neurosurg Focus. 1998, 4:e1.

26. Kothbauer K, Deletis V, Epstein FJ: Intraoperative spinal cord monitoring for intramedullary surgery: an essential adjunct. Pediatr Neurosurg. 1997, 26:247-54. 10.1159/000121199

27. Acharya S, Palukuri N, Gupta P, Kohli M: Transcranial motor evoked potentials during spinal deformity corrections-safety, efficacy, limitations, and the role of a checklist. Front Surg. 2017, 4:8. 10.3389/fsurg.2017.00008

28. Vitale MG, Skaggs DL, Pace GI, et al.: Best practices in intraoperative neuromonitoring in spine deformity surgery: development of an intraoperative checklist to optimize response. Spine Deform. 2014, 2:333-339. 10.1016/j.jspd.2014.05.003 